\title{
Artificial Neural Networks for rock and soil cutting slopes stability condition prediction
}

\author{
Joaquim Tinoco ${ }^{1,2}, \mathrm{PhD}$; António Gomes Correia ${ }^{1}, \mathrm{PhD}$; Paulo Cortez ${ }^{2}, \mathrm{PhD}$ and \\ David Toll ${ }^{3}, \mathrm{PhD}$
}

${ }^{1}$ ISISE - Institute for Sustainability and Innovation in Structural Engineering, School of Engineering, University of Minho, Guimarães, Portugal, jtinoco@ civil.uminho.pt.

${ }^{2}$ ALGORITMI Research Center/Department of Information Systems, School of Engineering, University of Minho, Guimarães, Portugal.

${ }^{3}$ School of Engineering and Computing Sciences, University of Durham, Durham, UK.

\begin{abstract}
This study aims to develop a tool able to help decision makers to find the best strategy for slopes management tasks. It is known that one of the main challenges nowadays for every developed or countries undergoing development is to keep operational under all conditions their transportations infrastructure. However, due to the network extension and increased budget constraints such challenge is even more difficult to accomplish. Keeping in mind the strong impact of a slope failure in the transportation infrastructure it is important to develop tools able to help minimizing this situation. Accordingly, and in order to achieve this goal, the high flexible learning capabilities of Artificial Neural Networks (ANNs) were applied in the development of a classification tool aiming to identify the stability condition of a rock and soil cutting slopes, keeping in mind the use of information usually collected during routine inspections activities (visual information) to feed them. For that, it was followed a nominal classification strategy and, in order to overcome the problem of imbalanced data, three training sampling approaches were explored: no resampling, SMOTE (Synthetic Minority Over-sampling Technique) and Oversampling. The achieved results are presented and discussed, comparing the achieved performance for both slope types (rock and soil cuttings) as well as the effect of the sampling approaches. An input-sensitivity analysis was applied, allowing to measure the relative influence of each model attribute.
\end{abstract}

\section{INTRODUCTION}

A nowadays challenge concerning to the transportation network, mainly for every developed or countries undergoing development that have invested and keep investing to build a safe and functional transportation network, is how to keep it operational under all conditions, keeping in mind its extension and the increased budget limitation for maintenance and repair tasks. Thus, taken into account the key importance of the transportation system in modern societies, it is fundamental to develop new tools able to help in its management. 
Keeping in mind the strong impact of a slope failure in the transportation infrastructure, namely for the railway it is important to develop tools able to help minimizing this situation.

Over time, several efforts have been made toward the development of a system to detect slope failures. However, most of the systems were developed for natural slopes, presenting some limitations when applied to engineered (human made) slopes. In addition, they have limited applicability as most of them were developed based on particular case studies or using small databases. Furthermore, another aspect that can limit its applicability is related with the information required to feed them, such as data taken from complex tests or from expensive monitoring systems. Pourkhosravani and Kalantari (2011) summarized in their work some of the current methods for slope failure detection, which were grouped into Limit Equilibrium (LE) methods, Numerical Analysis methods, Artificial Neural Networks and Limit Analysis methods. There are also approaches based on finite elements methods (Suchomel et al. 2010), reliability analysis (Sivakumar and Murthy, 2005; Husein Malkawi et al. 2000), as well as some methods making use of data mining (DM) algorithms (Cheng and Hoang 2016, Ahangar-Asr et al. 2010, Yao et al. 2008, Kang et al., 2017; Kang and Li, 2016; Suman et al. 2016). More recently, a new flexible statistical system was proposed by Pinheiro et al. (2015), based on the assessment of different factors that affect the behavior of a given slope, which are weighted in order to calculate a final indicator of the slope stability condition.

In summary, most of the approaches so far proposed share the main limitations, which are related with its applicability domain or dependency on information that is difficult to obtain. In fact, the assessment of the stability condition of given slope is a multivariable problem characterized by a high dimensionality.

Artificial Neural Networks (ANNs) are one of the most well known Data Mining (DM) algorithms, which have been applied with success in different knowledge domains, such as web search, spam filters, recommender systems, and fraud detection (Domingos 2012). Also in civil engineering field, several applications can be found. For example, ANNs were applied in the study of physical and mechanical properties of jet grouting columns (Tinoco et al. 2014, Tinoco et al. 2016). Indeed, the high learning capabilities of this algorithm give it the ability to model complex nonlinear mappings. Thus, in this work the high flexible learning capabilities of Artificial Neural Networks (ANNs) were used to predict the stability condition of rock and soil cutting slopes according to a pre-defined classification scale based on four levels (classes). Moreover, one of the underlying premises of this work is to use only information that can be easily obtained. For that, more than fifty variables related with data collected during routine inspections as well as geometric, geological and geographic data were used to feed the models. With this methodology it is intended to identify critical zones for which more detailed information can then be obtained in order to perform more detailed stability analysis. Such novel approach is intended to support railway network management companies to allocate the available funds to the priority assets according to their stability condition. 


\section{METHODOLOGY}

\section{Data characterization}

To fit the proposed models for stability condition identification, from this point referred to as EHC (Earthwork Hazard Category (Power et al. 2016)), of rock and soil cutting slopes two database were compiled respectively. Both databases, containing information collected during routine inspections and complemented with geometric, geological and geographic data of each slope, were gathered by Network Rail workers and are concerned with the railway network of the UK. For each slope a class of the EHC system was defined by the Network Rail Engineers based on their experience/algorithms (Power et al. 2016), which will be assumed as a proxy for the real stability condition of the slope for year 2015. The EHC system comprises 4 classes ${ }^{1}$ ("A", "B", "C" and "D") where "A" represents a good stability condition and "D" a bad stability condition. In other words, the expected probability of failure is higher for class " $D$ " and lower for class " $A$ ".

Both databases contain a significant number of records. The rock slopes database comprises 5945 records, while the soil cutting slopes database is bigger, having 10928 records available. FIG. 1 plots the distribution of EHC classes for each database. From their analysis, it is possible to observe a high asymmetric distribution (imbalanced data), particularly for the rock cutting slopes database. In fact, more than $86 \%$ of the rock slopes are classified as "A". Although this type of asymmetric distribution, where most of the slopes present a low probability of failure (class "A"), is normal and desirable from the safety point of view and slope network management, it can represent an important challenge for data-driven models learning, as detailed in next section. The proposed models for EHC identification of rock and soil cutting slopes were fed with more than fifty variables normally collected during routine inspections and complemented with geometric, geographic and geological information. To be precise, 65 variables were used in the rock slopes study and 51 variables in soil cutting slopes. Since the number of analyzed variables is high (65/51), just a few examples of the variables used to feed the models are here enumerated: height, slope angle, presence of rock outcrops, animal activity, presence of boulders, ground cover, rock type, dangerous trees, number of root balls, rock strength, etc.

\section{Modeling}

In this work, ANNs were trained to predict EHC of rock and soil cutting slopes. ANNs, although not new, they are supported in a strong background and experience. Indeed, they have been applied in the past with high success in different knowledge domains including in civil engineering (Chou et al. 2016, Gomes Correia et al. 2013). There are also some examples of ANNs applications in slope stability analysis (Wang et al. 2005, Cheng et al. 2012).

ANNs are learning machines that were initially inspired in functioning of the human brain (Keniget et al. 2001). The information is processed using iteration among several neurons. This technique is capable of modeling complex non-linear mappings and is

\footnotetext{
${ }^{1}$ The original EHC system comprised 5 levels (A, B, C, D and E) (Power et al. 2016). However, and due to the reduced number of slopes classified as E, classes D and E were combined in one, named as D.
} 
robust in exploration of data with noise. In this study we adopt the multilayer perceptron that contains only feedforward connections, with one hidden layer containing $H$ processing units. Because the ANN performance is sensitive to $H$ (a trade-off between fitting accuracy and generalization capability), a grid search of $\{0$; $2 ; 4 ; 6 ; 8\}$ was adopted under an internal (i.e. applied over training data) three fold cross validation during the learning phase to find the best $H$ value. Under this grid search, the $H$ value that produced the lowest MAE (Mean Absolute Error) was selected, and then the ANN was retrained with all of the training data. The neural function of the hidden nodes was set to the popular logistic function $1 /\left(1+\mathrm{e}^{-\mathrm{x}}\right)$.

The problem of EHC determination was approached following a nominal classification strategy, where the network calculates a probability for each EHC level and then is selected the class corresponding to the highest probability.

In addition, and in order to minimize the effect of the imbalanced data (see FIG. 1), Oversampling (Ling and Li 1998) and SMOTE (Synthetic Minority Oversampling Technique) (Chawla et al. 2002) approaches were applied over the training data before fitting the models. When approaching imbalanced classification tasks, where there is at least one target class label with a smaller number of training samples when compared with other target class labels, the simple use of a soft computing training algorithm will lead to data-driven models with better prediction accuracies for the majority classes and worst classification accuracies for the minority classes. Thus, techniques that adjust the training data in order to balance the output class labels, such as Oversampling and SMOTE, are commonly used with imbalanced datasets. In particular, Oversampling is a simple technique that randomly adds samples (with repetition) of the minority classes to the training data, such that the final training set is balanced. SMOTE is a more sophisticated technique that creates "new data" by looking at nearest neighbors to establish a neighborhood and then sampling from within that neighborhood. It operates on the assumptions that the original data is similar because of proximity. We note that the different sampling approaches were applied only to training data, used to fit the data-driven models, and the test data (as provided by the 5 -fold procedure) was kept without any change.

For models evaluation and comparison, three classification metrics were adopted: recall, precision and $F_{1 \text {-score }}$ (Hastie et al. 2009). The recall measures the ratio of how many cases of a certain class were properly captured by the model. In other words, the recall of a certain class is given by TruePositives/(TruePositives + FalseNegatives). On the other hand, the precision measures the correctness of the model when it predicts a certain class. More specifically, the precision of a certain class is given by TruePositives/(TruePositives + FalsePositives). The $F_{1-\text { score }}$ was also calculated, which represent a trade-off between the recall and precision of a class. The $F_{1 \text {-score }}$ correspond to the harmonic mean of precision and recall, according to the following expression:

$$
F_{1-\text { score }}=2 \cdot \frac{\text { precision } \cdot \text { recall }}{\text { precision }+ \text { recall }}
$$

For all three metrics, the higher the value, the better are the predictions, ranging from 
$0 \%$ to $100 \%$.

The generalization capacity of the models was accessed through a 5-fold crossvalidation approach under 20 runs (Hastie et al. 2009). This means that each modeling setup is trained $5 \times 10=100$ times. Also, the three prediction metrics are always computed on test unseen data (as provided by the 5 -fold validation procedure).

All experiments were conducted under the R statistical environment ( $R$ Team 2009). ANN algorithm was trained using the rminer package (Cortez 2010), which facilitates its implementation, as well as different validation approaches such as the crossvalidation adopted in this work.

\section{RESULTS AND DISCUSSION}

Following are presented and discussed the achieved performance in EHC determination of both rock and soil cutting slopes based on ANN algorithm, as well as the effect of the three training sampling approaches explored: Normal (no resampling), OVERed (Oversampling) and SMOTEd (SMOTE).

FIG. 2 gives an overview of ANNs models performance in rock and soil cutting slopes, based on recall, precision and F1-score.

Concerning to rock cutting slopes study (left side of FIG. 2) the achieved performance is somewhat lower independently of the resampling approach applied. Although a very high performance is observed for class "A" $\left(F_{1-\text { score }}\right.$ higher than $\left.95 \%\right)$, for class "C" and particularly for class " $D$ ", all models evidence clear difficulties in predicting such classes correctly. In fact, and using $F_{1 \text {-score }}$ as reference, the best performance in identification of slopes of class "D" is lower than 14\% (see FIG. 2) which was achieved by applying SMOTE resampling approach. From FIG. 3a analysis, which plots the relation between observed and predicted EHC values of rock cutting slopes by applying oversampling approach (best overall fit), it is clear the model difficulties in correctly predicting class " $C$ " and particularly class " $D$ ", for which the expected probability of failure is higher. As shown, only around $12 \%$ of rock cutting slopes classified as " $D$ " are correctly identified, which represents a low performance, namely when compared with soil cutting slopes study as following discussed. Overall, these results show that the methodology applied for EHC determination of rock cutting slopes needs future development in order to overcome this gap.

Relating to soil cutting slopes study, a very promising performance is observed, as shown in FIG. 2 (right side). For example, soil cutting slopes of class "A" can be correctly identified, either with or without resampling. Also for classes "B" and "C" a promising performance is also observed, with an $F_{1-\text { score }}$ around $55 \%$. Concerning the class "D", although an $F_{1-\text { score }}$ lower than $36 \%$ was achieved, the obtained value for recall metric around 57\% shows a good performance for class " $\mathrm{D}$ " identification. Analyzing FIG. $3 \mathrm{~b}$ that shows the relation between observed and predicted EHC values according to the best overall fit (SMOTEd), one can see that the model performance is very promising. Indeed, when a SMOTE resampling approach is applied, ANN algorithm is able to predict correctly around $57 \%$ of soil cutting slopes of class " $\mathrm{D}$ ", which represents a very promising performance if we take into account that this is the minority class. For class " $\mathrm{C}$ ", although the accuracy is not so high (around 40\%), when not predicted as " $\mathrm{C}$ " they are classified as belonging to the 
closest class, that is, "B" or " $D$ ". This type of misclassification is also observed for classes "A", "B" and "D", which can be interpreted as positive point. Concerning to classes "A" and "B", the ANN model was able to identify it very accurately.

Comparing the achieved results of rock and soil cutting slopes studies, the proposed models for soil cutting slopes are more effective, namely in the identification of classes " $C$ " and "D" for which the probability of failure is higher (see FIG. 2). A possible explanation for the lower performance, namely for classes " $C$ " and " $D$ " of rock cutting slopes could be related with the EHC classes being assumed as representative of the real stability condition of each slope. Indeed, analyzing the number of slope failures by EHC class for rock slopes there are some indications that the classification attributed to each rock slope could lack of some accuracy as reported in the work of Power et al. (2016), which used the same source of information. It would be expected that most of the failures would occur in slopes of classes " $C$ " and mainly "D". However, for rock slopes such behavior is not observed as reported on Power et al. (2016). In fact, the number of failures for each EHC class is almost constant from classes "A" to "D", particularly when compared with soil cuttings. For example, the number of failures observed in rock cutting slopes of class " $\mathrm{C}$ " is only twice higher when compared to class "A". This observation shows that the defined classes for rock slopes have a poor correlation with actual failures.

These results show that a deeper data analysis is required, particularly in the study of rock cutting slopes. For example, the number of variables taken as model attributes might be too high and may be influencing the generalization performance of the models.

Thus, and in order to better understanding the proposed models, the relative importance of each model input was measured based on a sensitive analysis. For that, the methodology proposed by Cortez and Embrechts (2013) was applied. Accordingly, FIG. 4 shows the relative importance of the 20 most relevant variables based on the two models that achieved the overall best performance in EHC determination of rock (left side) and soil (right side) cutting slopes (see FIG. 3). From their analysis, it is observed that the height of the slope is one of the most relevant variables affecting the slope stability condition, either for rock or soil cutting slopes. On the other hand, and particularly for rock slopes, it is observed that the first seven most relevant variables are responsible for around $70 \%$ of the total influence.

These results, together with achieved performance, suggest a need of revision of the variables used as model attributes. Accordingly, and as a future works, it is intended to apply a more sophisticated feature selection method in order to improve models performance. In particular, it will be explored a multi-objective evolutionary computation method that simultaneously maximizes prediction performance and minimizes the number of inputs used.

As a final observation, and considering the overall performance of all models, it should be underlined the potential of ANNs, or even other soft computing algorithms, in EHC determination of rock and soil cutting slopes. 


\section{ACKNOWLEDGMENTS}

This work was supported by FCT - "Fundação para a Ciência e a Tecnologia", within ISISE, project UID/ECI/04029/2013 as well Project Scope: UID/CEC/00319/2013 and through the post-doctoral Grant fellowship with reference SFRH/BPD/94792/2013. This work was also partly financed by FEDER funds through the Competitivity Factors Operational Programme - COMPETE and by national funds through FCT within the scope of the project POCI-01-0145-FEDER-007633. This work has been also supported by COMPETE: POCI-01-0145-FEDER-007043. A special thanks goes to Network Rail that kindly made available the data (basic earthworks examination data and the Earthworks Hazard Condition scores) used in this work.

\section{REFERENCES}

Ahangar-Asr, A., Faramarzi, A. and Javadi, A. (2010). A new approach for prediction of the stability of soil and rock slopes. Engineering Computations, 27(7), 878-893.

Chawla, N., Bowyer, K., Hall, L. and Kegelmeyer, W. (2002). Smote: synthetic minority oversampling technique. Journal of artificial intelligence research, 32, 321-357.

Cheng, M. and Hoang, N. (2016). Slope collapse prediction using bayesian framework with k-nearest neighbor density estimation: Case study in taiwan. Journal of Computing in Civil Engineering, 30(1), 04014116.

Cheng, M., Roy, A. and Chen, K. (2012). Evolutionary risk preference inference model using fuzzy support vector machine for road slope collapse prediction. Expert Systems with Applications, 39(2), 1737-1746.

Chou, J., Yang, K. and Lin, J. (2016). Peak shear strength of discrete fiber-reinforced soils computed by machine learning and metaensemble methods. Journal of Computing in Civil Engineering, 04016036.

Cortez, P. (2010). Data mining with neural networks and support vector machines using the r/rminer tool. In P. Perner (Ed.), Advances in Data Mining: Applications and Theoretical Aspects, 10th Industrial Conference on Data Mining, Berlin, Germany, pp. 572-583. LNAI 6171, Springer.

Cortez, P. and Embrechts, M. (2013). Using sensitivity analysis and visualization techniques to open black box data mining models. Information Sciences, 225, 1-17.

Domingos, P. (2012). A few useful things to know about machine learning. Communications of the ACM, 55(10), 78-87.

Gomes Correia, A., Cortez, P., Tinoco, J. and Marques, R. (2013). Artificial intelligence applications in transportation geotechnics. Geotechnical and Geological Engineering, 31(3), 861-879.

Hastie, T., Tibshirani, R. and Friedman, J. (2009). The Elements of Statistical Learning: Data Mining, Inference, and Prediction (Second Edition ed.). SpringerVerlag New York.

Husein Malkawi, A., Hassan, W. and Abdulla, F. (2000). Uncertainty and reliability analysis applied to slope stability. Structural Safety, 22(2), 161-187.

Kang, F., and Li, J. (2016). Artificial bee colony algorithm optimized support vector regression for system reliability analysis of slopes. Journal of Computing in Civil 
Engineering, 04015040.

Kang, F., Li, J. S., Wang, Y., and Li, J. (2017). Extreme learning machine based surrogate model for analyzing system reliability of soil slopes. European Journal of Environmental and Civil Engineering, 21(11), 1341-1362.

Kenig, S., Ben-David, A., Omer, M. and Sadeh, A. (2001). Control of properties in injection molding by neural networks. Engineering Applications of Artificial Intelligence, 14(6), 819-823.

Ling, C. and Li, C. (1998). Data mining for direct marketing: Problems and solutions. In KDD, 98, 73-79.

Pinheiro, M., Sanches, S., Miranda, T., Neves, A., Tinoco, J., Ferreira, A. and Gomes Correia, A. (2015). A new empirical system for rock slope stability analysis in exploitation stage. International Journal of Rock Mechanics and Mining Sciences, 76, 182-191.

Pourkhosravani, A. and Kalantari, B. (2011). A review of current methods for slope stability evaluation. Electronic Journal of Geotechnical Engineering, 16.

Power, C., Mian, J., Spink, T., Abbott, S. and Edwards, M. (2016). Development of an evidence-based geotechnical asset management policy for network rail, great britain. Procedia Engineering, 143, 726-733.

Sivakumar Babu, G., and Murthy, D. (2005). Reliability analysis of unsaturated soil slopes. Journal of Geotechnical and Geoenvironmental Engineering, 1423-1428.

Suchomel, R. et al. (2010). Comparison of different probabilistic methods for predicting stability of a slope in spatially variable $c^{\prime}$ soil. Computers and Geotechnics, 37(1), 132-140.

Suman, S., Khan, S., Das, S., and Chand, S. (2016). Slope stability analysis using artificial intelligence techniques. Natural Hazards, 84(2), 727-748.

Team, R. (2009). R: A language and environment for statistical computing. R Foundation for Statistical Computing, Viena, Austria. Web site: http://www.rproject.org/.

Tinoco, J., Gomes Correia, A. and Cortez, P. (2014). Support vector machines applied to uniaxial compressive strength prediction of jet grouting columns. Computers and Geotechnics, 55, 132-140.

Tinoco, J., Gomes Correia, A. and Cortez, P. (2016). Jet grouting column diameter prediction based on a datadriven approach. European Journal of Environmental and Civil Engineering, 1-22.

Wang, H., Xu, W. and $\mathrm{Xu}, \mathrm{R}$. (2005). Slope stability evaluation using back propagation neural networks. Engineering Geology, 80(3), 302-315.

Yao, X., Tham, L. and Dai, F. (2008). Landslide susceptibility mapping based on support vector machine: a case study on natural slopes of hong kong, china. Geomorphology, 101(4), 572-582. 


\section{FIGURES}

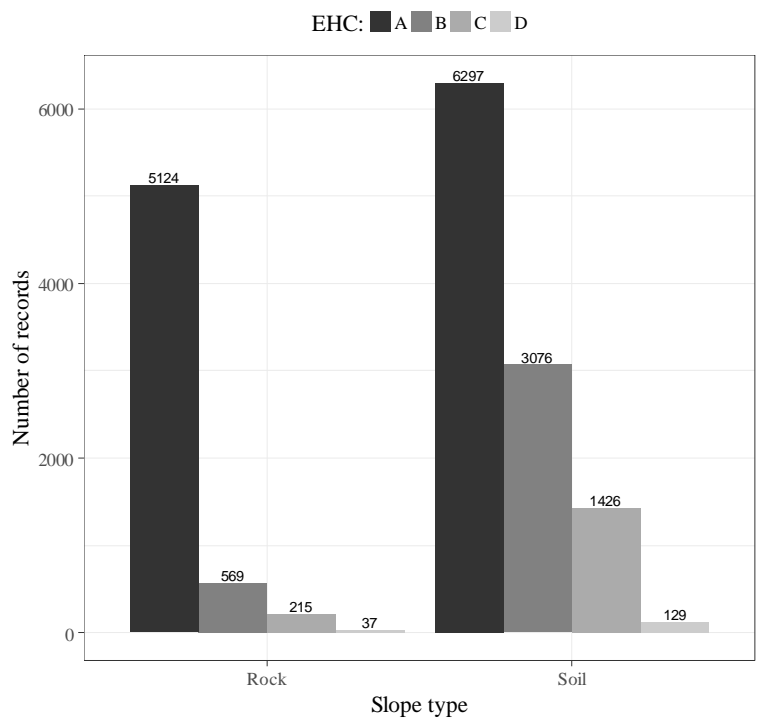

FIG. 1. Rock and soil cutting slopes data distribution by EHC classes

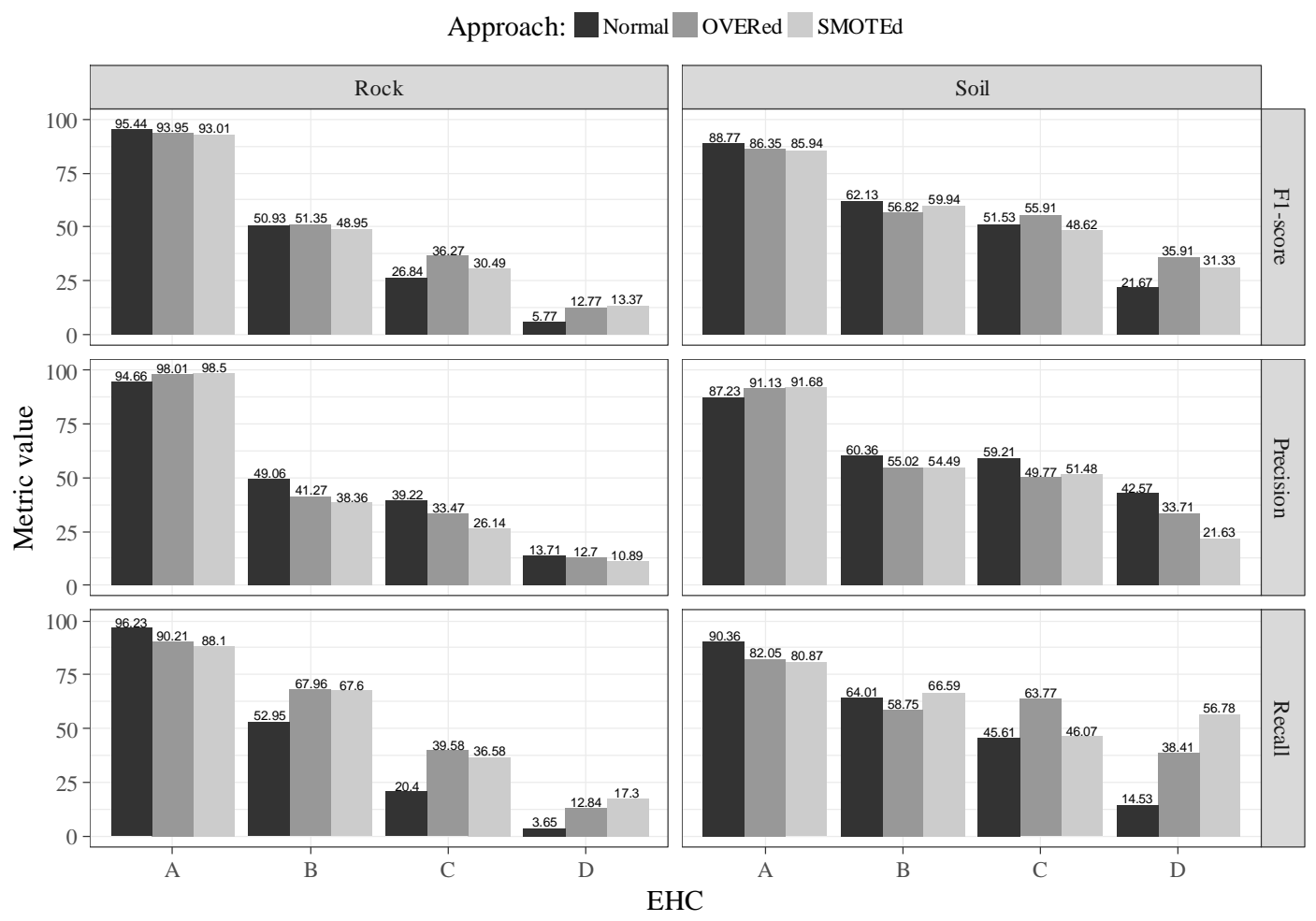

FIG. 2. ANN performance comparison in EHC determination of both rock and soil cutting slopes based on recall, precision and F1-score 

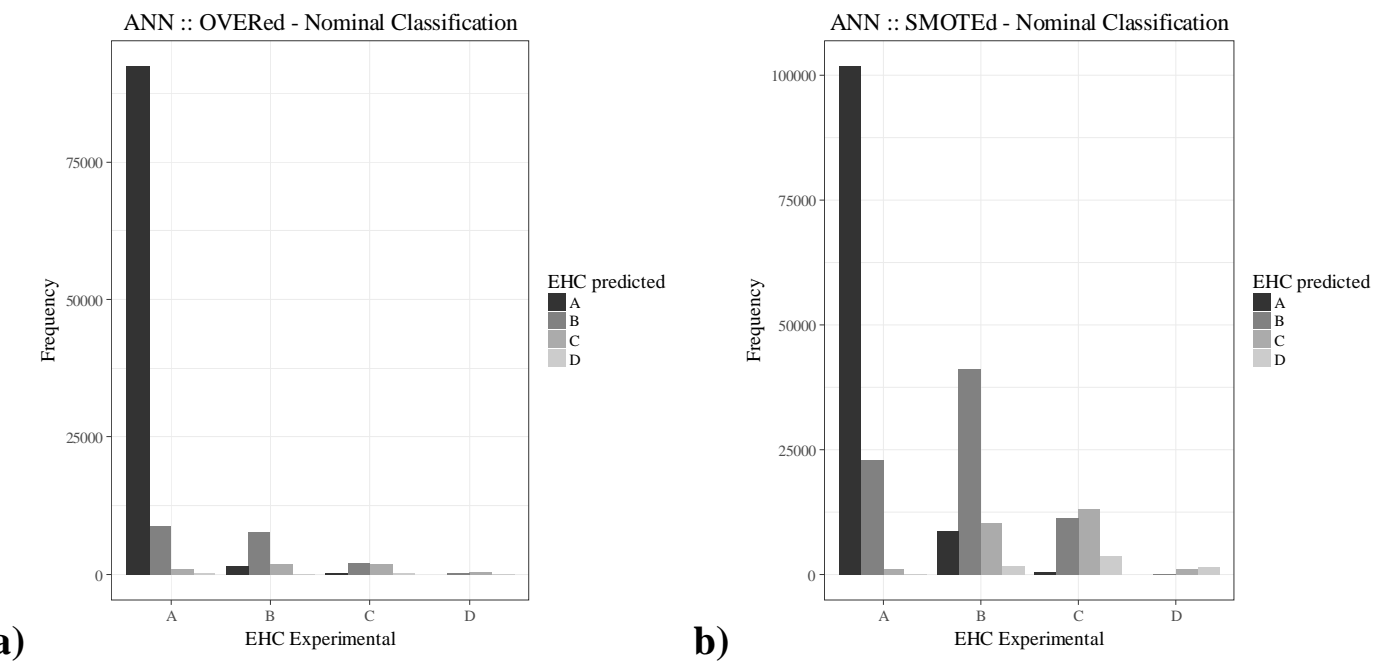

FIG. 3. ANN performance: a) Rock cutting slopes following an OVERed approach; b) Soil cutting slopes following a SMOTEd approach
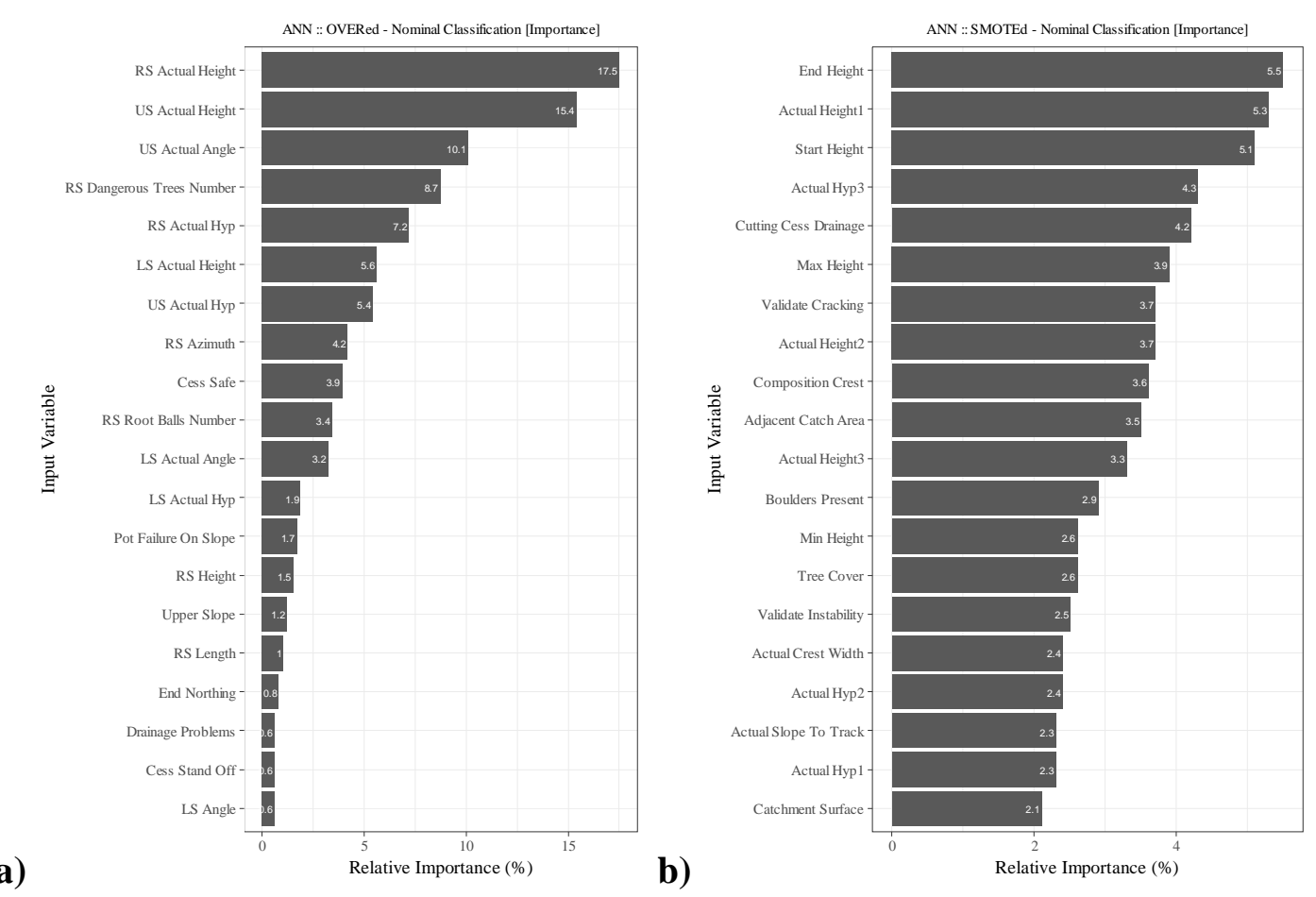

FIG. 4. Relative importance bar plot of the 20 most relevant variables according to ANN models in EHC determination: a) Rock cutting slopes following an OVERed approach; b) Soil cutting slopes following a SMOTEd approach 